\title{
Virtual design method for controlled failure in foldcore sandwich panels
}

\author{
R. Sturm $\left.{ }^{*}\right)^{1)} \cdot$ S. Fischer ${ }^{2)}$ \\ 1) Institute of Structures and Design, German Aerospace Center (DLR), Germany \\ 2) Institute of Aircraft Design, University of Stuttgart, Germany
}

Keywords: crash, explicit simulation, sandwich structures, foldcore, energy absorption

\begin{abstract}
For certification, novel fuselage concepts have to prove equivalent crashworthiness standards compared to the existing metal reference design. Due to the brittle failure behaviour of CFRP this requirement can only be fulfilled by a controlled progressive crash kinematics. Experiments showed that the failure of a twin-walled fuselage panel can be controlled by a local modification of the core through-thickness compression strength. For folded cores the required change in core properties can be integrated by a modification of the fold pattern. However, the complexity of folded cores requires a virtual design methodology for tailoring the fold pattern according to all static and crash relevant requirements. In this context a foldcore micromodel simulation method is presented to identify the structural response of a twin-walled fuselage panels with folded core under crash relevant loading condition. The simulations showed that a high degree of correlation is required before simulation can replace expensive testing. In the presented studies, the necessary correlation quality could only be obtained by including imperfections of the core material in the micromodel simulation approach.
\end{abstract}

\section{Introduction}

Sandwich structures with composite facesheets are commonly recognized as a promising concept for structural airframe design due to their excellent stiffness-to-weight ratio. However, until now the application of sandwich structures is limited to secondary structures, since further understanding is needed in the field of manufacturing, repair, vulnerability and safety before sandwich design can be applied on primary structures. One challenge for novel fuselage concepts is the required proof of equivalent safety standards with regard to the conventional fuselage design. Brittle failure mechanisms of CFRP (Carbon-fiber-reinforced polymer) structures make the verification of equivalent crashworthiness for CFRP fuselage concepts challenging, since conventional metal fuselages absorb a significant portion of the kinetic impact energy by plasticization of the frame structures.

Different strategies were identified for composite fuselages in various research programs to improve the energy absorption characteristics during crash incidents. The most common energy absorption strategy is the application of composite crushing [1-2]. Research projects addressing this topic identified further lightweight potential for a crashworthy CFRP fuselage design by absorbing the impact energy by a progressive failure of structural components. In this approach frames have to absorb sufficient energy during bending failure in a progressive predefined manner. However, all structural modifications to obtain a robust crash kinematics with survivable acceleration loads have to comply with the static requirements. Therefore, so called "kinematic hinges" have to be developed which enable a controlled frame failure with sufficient energy absorption capacity in the post failure domain under bending loads. While the specific energy absorption of composite 
structures can exceed metal under compression, the brittle failure mode under bending loads limits their energy absorption capacity during bending failure. Crash studies highlighted the importance of the installation of kinematic hinges for a crashworthy CFRP fuselage concept; especially if the vertical acceleration loads of the passengers should not exceed values obtained for the metallic reference design [3-4].

Whilst experimental and numerical studies were already performed on CFRP frame segments [5-7], there is a limited understanding of the failure mechanisms of CFRP sandwich panels under crash relevant bending loads. In this scope the presented study contributes to the assessment of the crashworthiness potential of twin-walled fuselage concepts. Foldcore is currently considered and investigated for future fuselage applications, since its open cellular design solves the problem of humidity accumulation of closed cellular sandwich cores like honeycombs [8-10]. It is a comparably novel core concept and can be produced out of different materials [11]. In the presented study only aramid foldcore is investigated. Besides the manufacturing and quality management, especially the simulation of folded core structures is challenging due to the complex damage behaviour of the paper material.

The folding process and the automation potentials are described by Klett [12]. Kärger et al. [13] investigated experimentally the failure of folded core under combined transverse compression and shear forces and compared stiffness and failure behaviour to honeycomb structures. Since the possibility of tailoring a fold pattern according to defined static requirements using experimental material characterisation programs is economically not feasible, simulation strategies are required to identify numerically the optimized core design. The material characterisation process and virtual determination of the material properties using both homogenised cores and core micromodels were investigated by Fischer [14]. Kilchert [15] and Sturm [16] analysed the high velocity impact resistance of foldcore sandwich panels and developed foldcore micromodels for use in an explicit FE code. Low velocity impact behaviour of twin-walled sandwich panels with folded cores is described by Heimbs [17] and Thomson et al. [18]. Mainly three types of modelling methodologies can be found in literature for modelling cellular sandwich cores made out of Aramid paper. Firstly, cellular core structures can be modelled using a homogeneous solid model representation [19,15]. The aramid paper can also be modelled using an elastic-plastic material description to simulate the instability failure in core micromodels [16]. The detailed composition of the aramid paper is considered by the multi-layered material description developed in the CELPACT project [15]. Hereby the different fibre and matrix distribution over the thickness is addressed by a multi-layered approach in which each layer is described by a different material and damage behaviour.

\section{Experimental study of a foldcore trigger mechanism for CFRP sandwich panels}

In experimental studies on controlled failure of sandwich panels under combined compression/bending loads, the functionality of a core trigger mechanism could be proven by the local adaptation of the core failure characteristics [20]. The principles of the core trigger concept are provided in Figure 1. As long as the shear stiffness remains within the required static envelope, the core trigger does not affect the structural behaviour of the fuselage panel. As soon as the loading condition of the panel exceeds a predefined load the locally weakened core fails at the trigger position. The core subsequently collapses. As the bending stiffness of the sandwich is significantly reduced at the position of failure, a kinematic hinge develops exactly at this location [21].

The versatility of foldcore offers the unique possibility to implement the core trigger by a local modification of the fold pattern. The fold pattern used in the experiment consists of a zig-zag pattern with additional integrated horizontal edges. Since folded cores fail due to buckling, the throughthickness compressive strength is affected if the fold pattern is modified. 


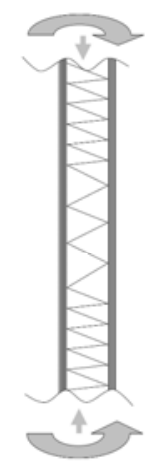

Static Envelope

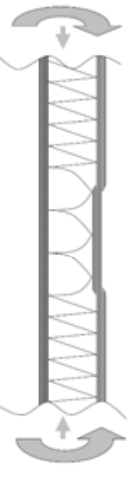

Core Collapse

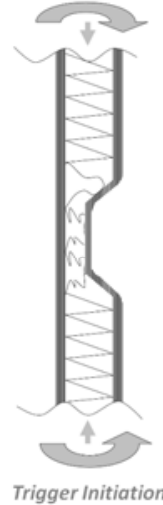

Trigger Initiation

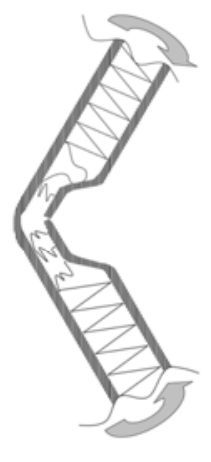

Hinge Development

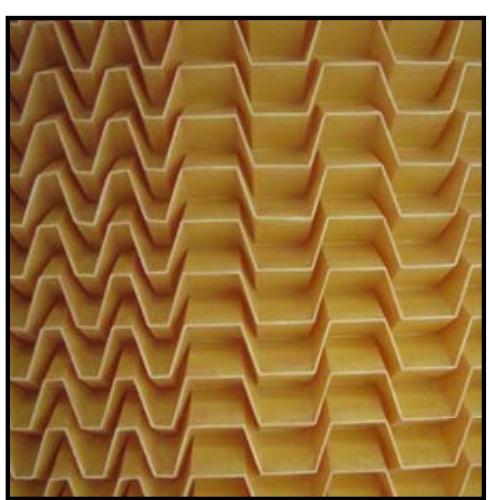

Fig. 1: Principles of the core trigger concept

Thus, the failure characteristics of the core can be adjusted by changing the length of the horizontal fold edge according to the hinge requirements. Since this modification can be directly implemented in the folding process, as shown in Figure 1, no splicing of different core structures is required. If a trigger mechanism needs to be investigated, it is beneficial to test the structural response under crash relevant loading condition. Especially a defined failure of the fuselage segment between the cargo crossbeams and the vertical struts is of particular interest to control the global crash kinematics. Studies investigating the crashworthiness of single aisle composite fuselage indicate a combination of bending and compression for this segment. To simplify the notation, the short description $A(\mathrm{~mm})$ is introduced for the ratio of bending $(\mathrm{kNmm})$ and compression loads $(\mathrm{kN})$.

For the investigated transport aircraft fuselage segment a representative loading condition with $A=$ $200 \mathrm{~mm}$ can be found in the literature [20,3]. Therefore, an experimental fixture for the investigation of the panel failure under crash relevant loading condition was developed.

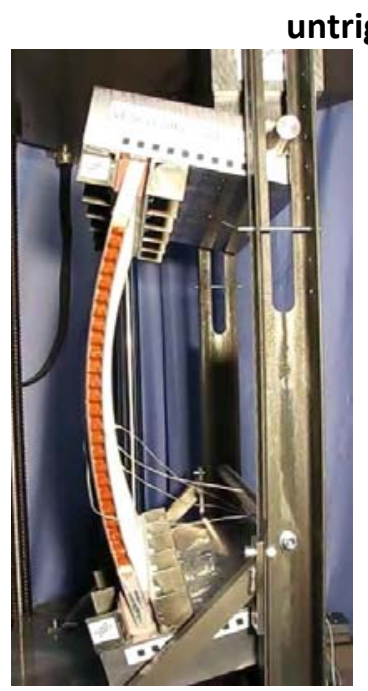

pre-failure untriggered

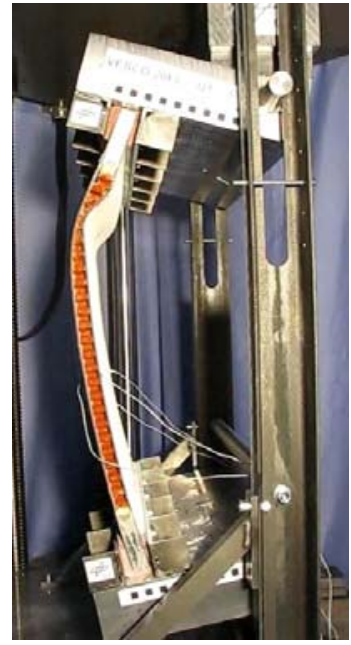

post-failure

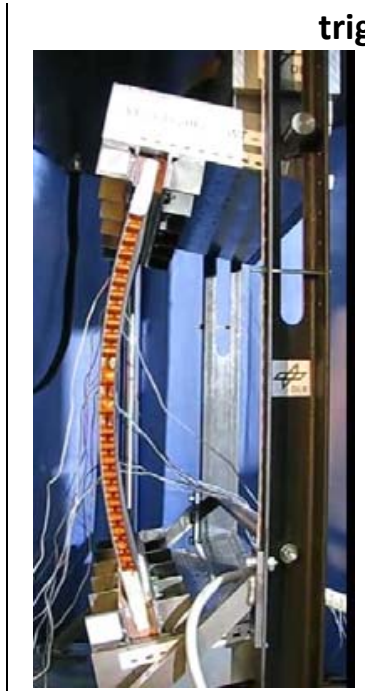

pre-failure triggered

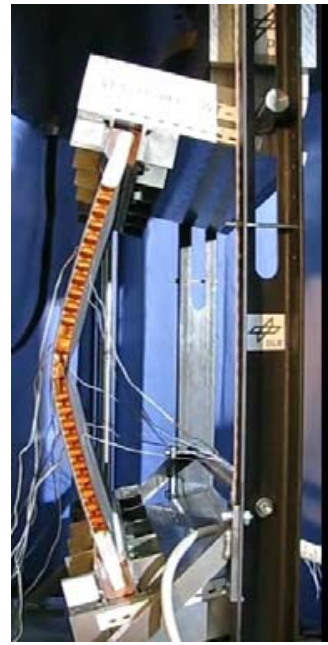

post-failure

Fig. 2: Verification of the core trigger concept by testing fuselage panels under a bending to compression ratio of $200 \mathrm{~mm}$ [20] 
For the validation of the core trigger two quasi-static tests under identical loading condition were conducted. The only difference was a local modified fold pattern for triggering. As shown in Figure 2, the position of failure could be controlled by the implemented core trigger. Whilst local stress concentrations close to the load introduction led to the failure of the untriggered sandwich panel, the position of failure could be altered by the integration of a core segment with a modified fold pattern.

The tests are only described briefly since the present paper focuses on numerical aspects and virtual testing. The experimental studies and the verification of the core trigger concept are published in detail [20].

\section{FE model for sandwich panels with foldcore micromodel}

For the experimental investigation large test specimens are required to introduce the combined loading. Since the structural response of folded cores is defined by its fold pattern, a numerical design approach is required to tailor the fold pattern according to all static- and crash-related requirements. As the material exhibits large non-linear behaviour during failure, an explicit FE code simulation with a detailed core micromodel seems favourable. In Figure 3 the main design features of the experimental fixture and their virtual representations are shown. Whilst for the elastic deformation of the leverage devices a coarse representation is sufficient, a detailed discretization of the test specimen is required to accurately model its failure modes. Initially simulation studies were performed to investigate a possible reduction of computational costs by using a multi-scaleapproach. Hereby the cellular structure of the core is modelled by homogenized solid elements outside the failure region. However, the simulation showed, that failure tended to be initiated by the interface between the detailed core micromodel and the homogenized core representation. Additionally, the missing symmetry of the fold core does not allow the application of symmetry boundary conditions. Therefore, a complete detailed core discretization was found to be necessary, especially if the exact position of failure is not known.

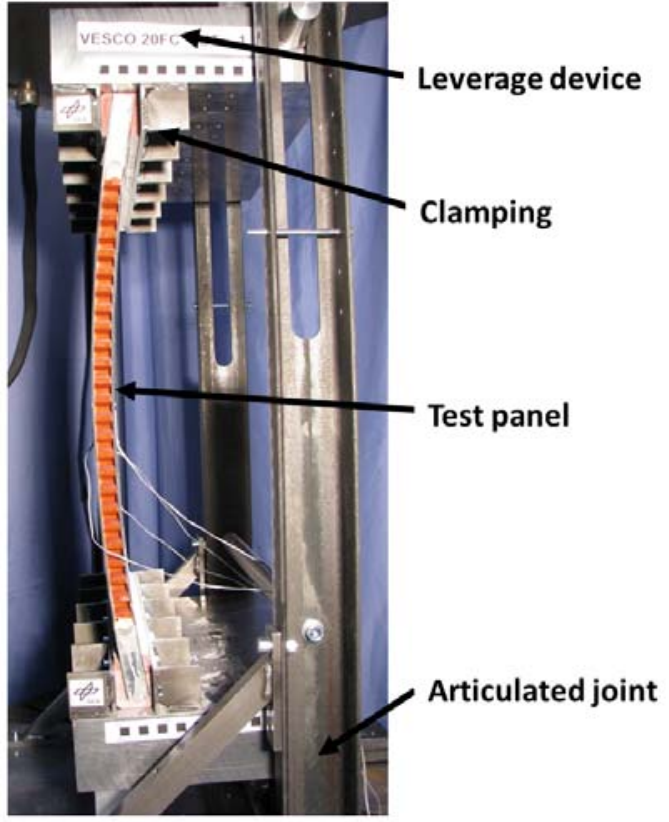

Test device for compression bending loading

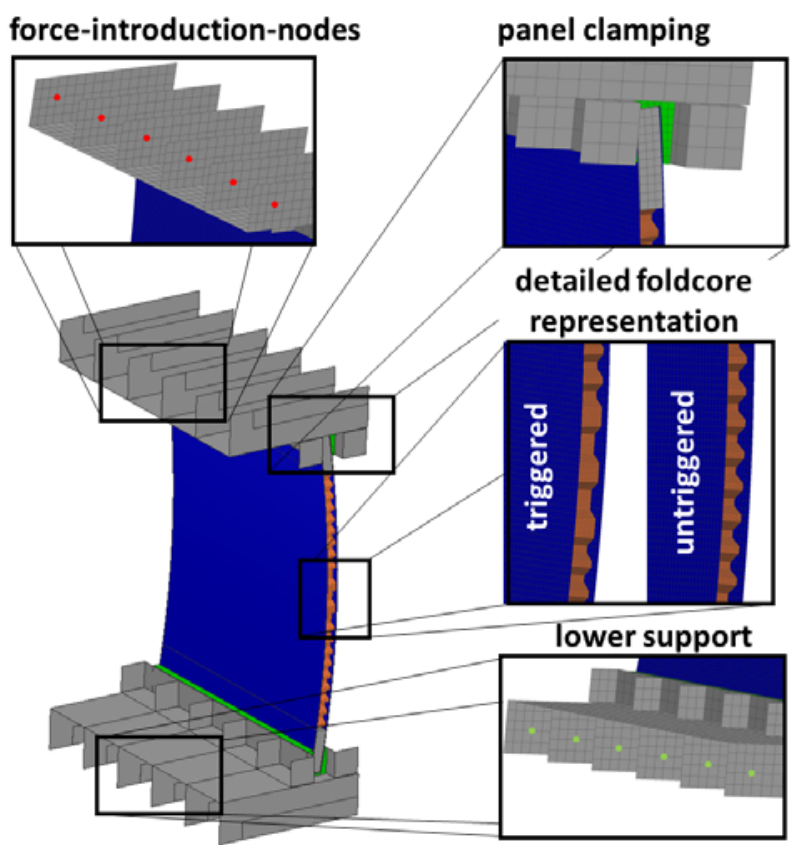

Numerical representation

Fig. 3: Discretization of the experimental test device 
The loading was introduced by displacement boundary conditions on structural nodes at the same position as in the experiment. Due to the explicit simulation approach the loading rate of the quasistatic tests had to be increased from $5 \mathrm{~mm} / \mathrm{min}$ to $1 \mathrm{~m} / \mathrm{s}$. By using a ramp-function the influence of inertia effects on the results could be damped to obtain a smooth loading condition during failure. Rotational nodal boundary conditions where applied on the nodes at the position of the lower support in the experiment.

The facesheets of the cylindrical sandwich panel with a radius of $2000 \mathrm{~mm}$ consisted of two identical Cytec HTS/977-2 prepreg laminates with the setup of $\left[90^{\circ} / 0^{\circ} / 90^{\circ} / 0^{\circ} / 0^{\circ} /+45^{\circ} /-45^{\circ} / 0^{\circ}\right]_{\mathrm{S}}$, where the $0^{\circ}$ direction corresponds to the flight direction. All FE simulations were performed with the explicit solver PAM-CRASH V.2009. The facesheets were modelled with the multi-layered composite material MAT 131 and the unidirectional composite global ply model (PLY 1) for the description of the prepreg ply [22]. To obtain the same loading as in the experiment, the load introduction is modelled in much detail. Thus, aluminium, resin inlay and clamping are modelled to represent the elastic flexibility of the experimental apparatus.

A methodology for the simulation of the aramid paper is applied for the core which was developed in the EU-funded project CELPACT. The used multi-layered modelling approach takes into account the notable variation of the mechanical properties in thickness direction due to inhomogeneous fibre distribution [23]. The material description, the extensive validation program and the numerical sensitivities are described in detail by Kilchert [15]. Especially the measurement of the foldcore through-thickness compressive properties was identified to be challenging since the paper fails by instabilities before the maximum compressive stress is reached. The usual approach to identify its compressive behaviour is the performance of virtual compression tests on a representative number of folded unit cells [11]. Sensitivity studies identified a slight mesh dependent structural response of the core. Hereby especially the failure peak stress is affected by the element size. An element size of $1 \mathrm{~mm}$ is recommended to restrict the mesh dependency [15]. The comparison between numerical simulation ( $1 \mathrm{~mm}$ element size) and the experiment of an aramid foldcore is provided in Figure 4 . The simulation shows a good agreement of the compressive stiffness and the crushing plateau. On the other hand the simulation overestimates the peak load before failure. However, correlation between various impact tests and simulation proved that a virtual assessment of the vulnerability against impact hazard is feasible for sandwich panels using the developed numerical foldcore representation. For the simulation of the panel failure under crash-relevant loading condition the multi-layered modelling approach was used for the description of the aramid paper. However, model size restricted the element size to $2 \mathrm{~mm}$ to reduce the computation time.
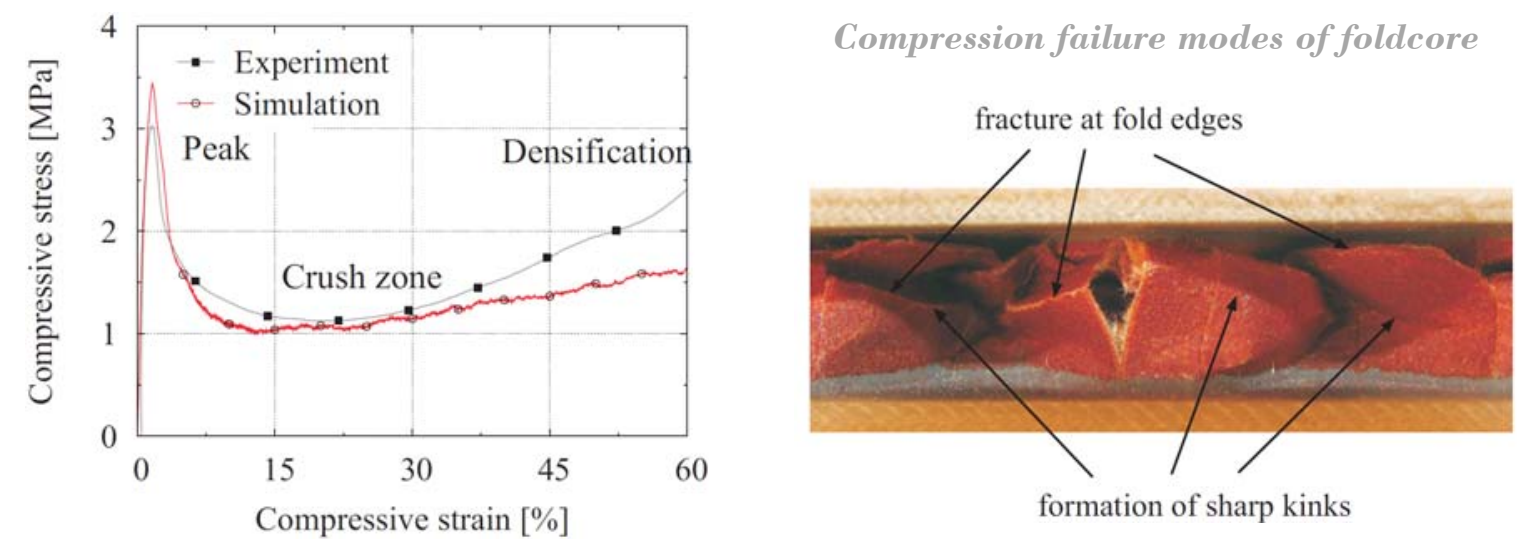

Fig. 4: Numerical correlation and identified failure mode of foldcore under compression failure [15] 
With the element size of $5 \mathrm{~mm}$ for the facesheets the complete FE-model consisted of 700000 elements. By using MPP (Massively Parallel Processing) calculation the computation time still allows virtual testing and the conduction of sensitivity studies. Since the fold patterns are based on simple geometric equations the parametric language of ANSYS-APDL was used for automated model generation.

For the validation of the simulation approach the experiments with a bending to compression ratio of $200 \mathrm{~mm}$ were simulated. The correlation between experiment and simulation is shown in Figure 5 . Hereby the strains were taken from the centreline of both facesheets and were plotted over the compressive force which was extracted from a section force output at the central panel position. Despite the used ramp function the inertia effects of the clamping recognizably influence the numerical output. For filtering the output and thus to exclude the dynamic effects from the numerical output, it was identified to be efficient to smooth the numerical output by a $3^{\text {rd }}$ degree polynomial function up to failure as shown in Figure 5.

The comparison shows, that although the elastic behaviour is accurately modelled, there is a significant discrepancy in the maximum failure load. For the untriggered panel the simulation even predicts a different failure mode. Instead of failing due to stress concentration at the loading introduction, the simulation predicts a compressive failure of the inner facesheet.
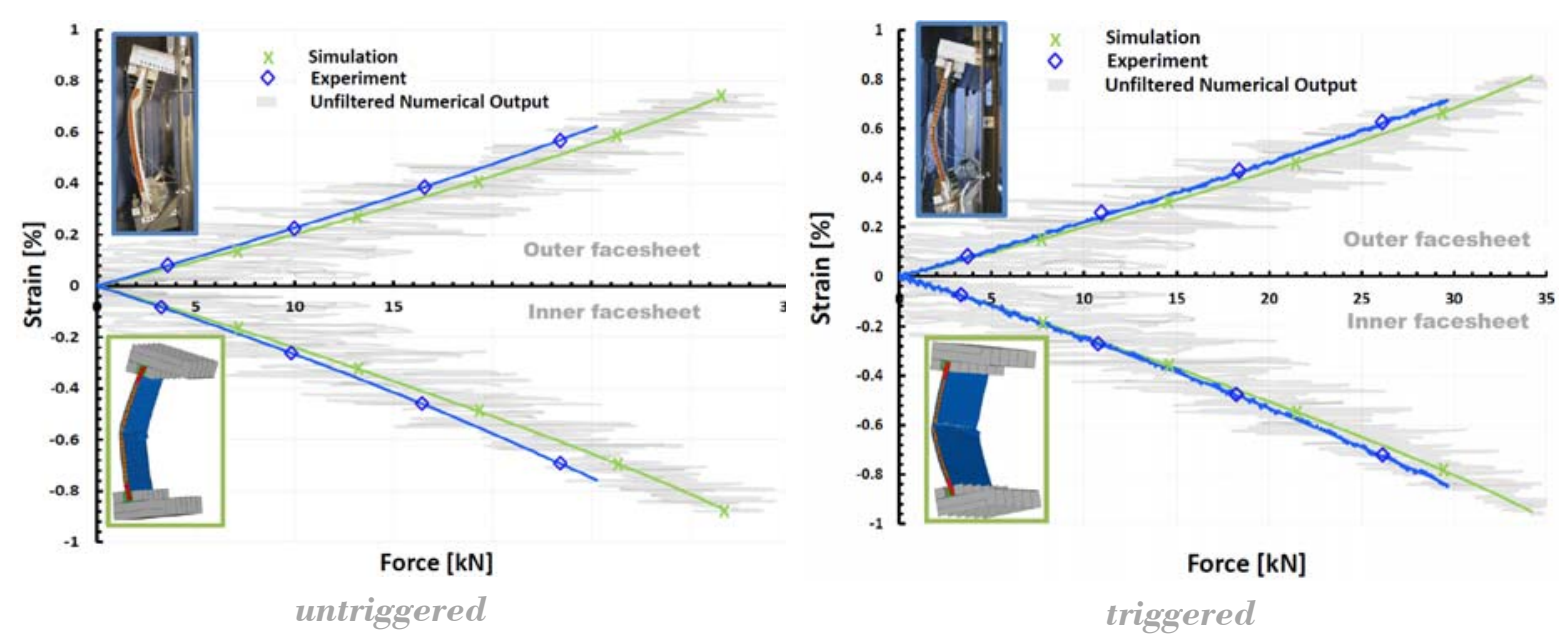

Fig. 5: Comparison of experimental and numerical strain-compression curve for the sandwich panel using the multi-layered modelling approach for the aramid paper $(A=200 \mathrm{~mm})$

The reason for this discrepancy is assumed to be based on the numerical overestimation of the core failure stresses. Unlike the simulation of impact damages, where the difference in the predicted peak load does not severely affect the results (since the overall energy absorption is modelled accurately) the inaccuracy alters the numerical result under crash loads. There is, therefore, a significant difference in the numerical damage assessment under impact and crash loads.

\section{Results and Discussions}

\subsection{Influence of the fold edges}

The comparison between simulation and experiment shows a delayed initiation of the core failure due to the numerical overestimation of the failure stress. Two reasons can be identified for this behaviour. On the one hand, the computation time does not allow the required small element size. Sensitivity studies showed a small increase of the compression failure load, if the mesh size is larger than $1 \mathrm{~mm}$ [15]. However, the main reason is assumed to originate in the idealised numerical micromodel of the foldcore. Detailed observations of the foldcore show distinct imperfections and irregularities of the aramid paper [14-15]. On the one hand in reality the resin layer consists of 
patches of irregularly distributed resin layers. The damaged fold edges are a second observed imperfection as depicted in Figure 6 . During the folding process the aramid fibres are pushed aside which reduces the local thickness of the main load carrying aramid layer. The effect of the weakened fold edge gets obvious if evaluating the failure mode of compressed aramid foldcore shown in Figure 4. The failure mode of ruptured fold edges, as found in the experiment, was not obtained in the numerical simulation $[15,20]$. However, a detailed representation of these irregularities is not feasible due to the numerical restrictions by the explicit simulation.

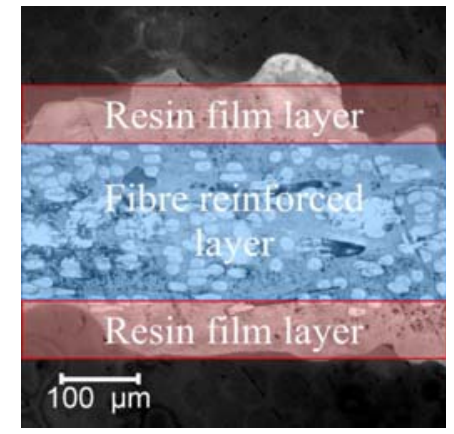

Paper material structure



Damage at the fold edges

Fig. 6: Material structure of the aramid paper at the undamaged region and at the folding edges [14] [15]

Whilst the core stiffness is mainly defined by its fold pattern and the material characteristics of the aramid paper, the instability failure of the core strongly depends on the nature of the fold edges. Simulation studies were performed to include the effect of the damaged fold edge in the simulation process without severely influencing the global structural response of the core within the elastic domain. The local degradation of the paper is assumed to be a pure reduction of the paper thickness at the fold edges due to the aramid fibres which are pushed aside during the manufacturing process. An extent of $1 \mathrm{~mm}$ for the weakened edge region was identified to be necessary to limit the computation cost. Figure 7 shows the unit cells for the untriggered and the triggered foldcore with the included degraded fold edges.
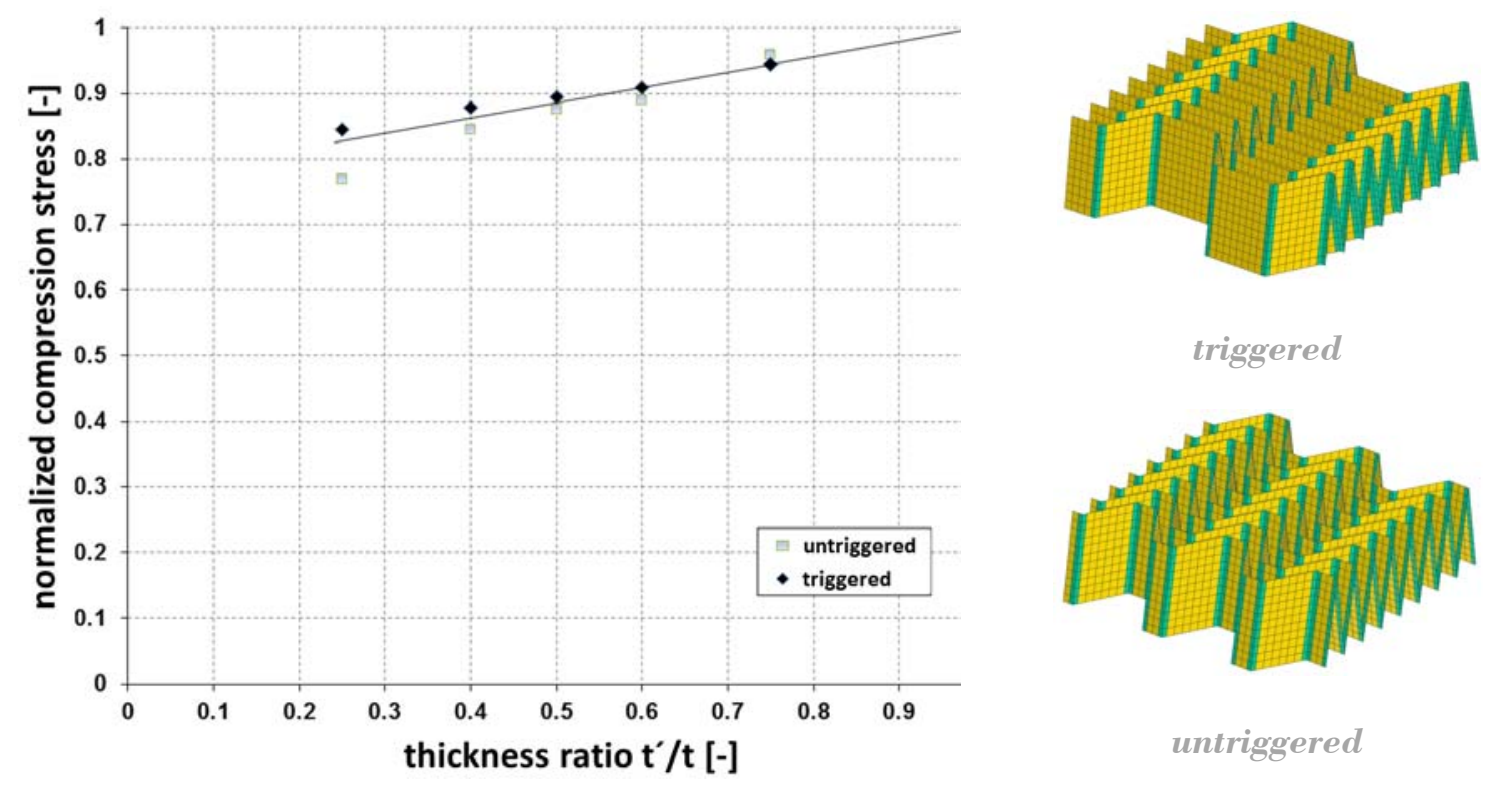

untriggered

Fig. 7: Numerical influence of damaged fold edges on the compression failure stress of the core 
Virtual compression tests on unit cells were performed to identify the effect of a reduced paper thickness at the fold edges. As shown in Figure 7 the simulation depicts an almost linear decrease of the failure load depending on the paper thickness. Hereby a similar normalised decline of failure stress is obtained for the triggered and the untriggered fold pattern. With the assumption that according to Figure 4 , the idealised representation of the foldcore is responsible for a $13 \%$ overestimation in failure load, a $50 \%$ reduction of paper thickness in the fold edges has to be assumed to obtain a similar failure stress as in the experiment. This reduction is realistic if considering the smallest fracture length through the load carrying fibre layer as highlighted in Figure 6.

Degraded fold edges with an extent of $1 \mathrm{~mm}$ were integrated for all fold edges in the simulation model. Despite an increase of elements from 700.000 to 830.000 elements and a reduction of the time step from $0.32 \mu \mathrm{s}$ to $0.23 \mu \mathrm{s}$ the computational time still allows the performance of virtual sensitivity studies.

\subsection{Validation of the numerical approach}

For comparability reasons the numerical model and the output parameters were kept identical except for integrating degraded fold edges. The correlation between experiment and simulation of the untriggered fuselage panel is shown in Figure $8(A=200 \mathrm{~mm})$. The integration of the degraded fold edges completely alters the predicted failure mechanism. Whilst in the simulation with the idealised foldcore representation a fracture of the inner facesheet was obtained (as shown in Figure 5), a core failure near the load introduction is predicted. Therefore, elastic deformation and failure mode shows a good correlation between simulation and experiment. Even the inner facesheet remained without fracture which corresponds to the experimental finding. Additionally the measured facesheet-strain over the cross-head loading is plotted for experiment and simulation in Figure 8.
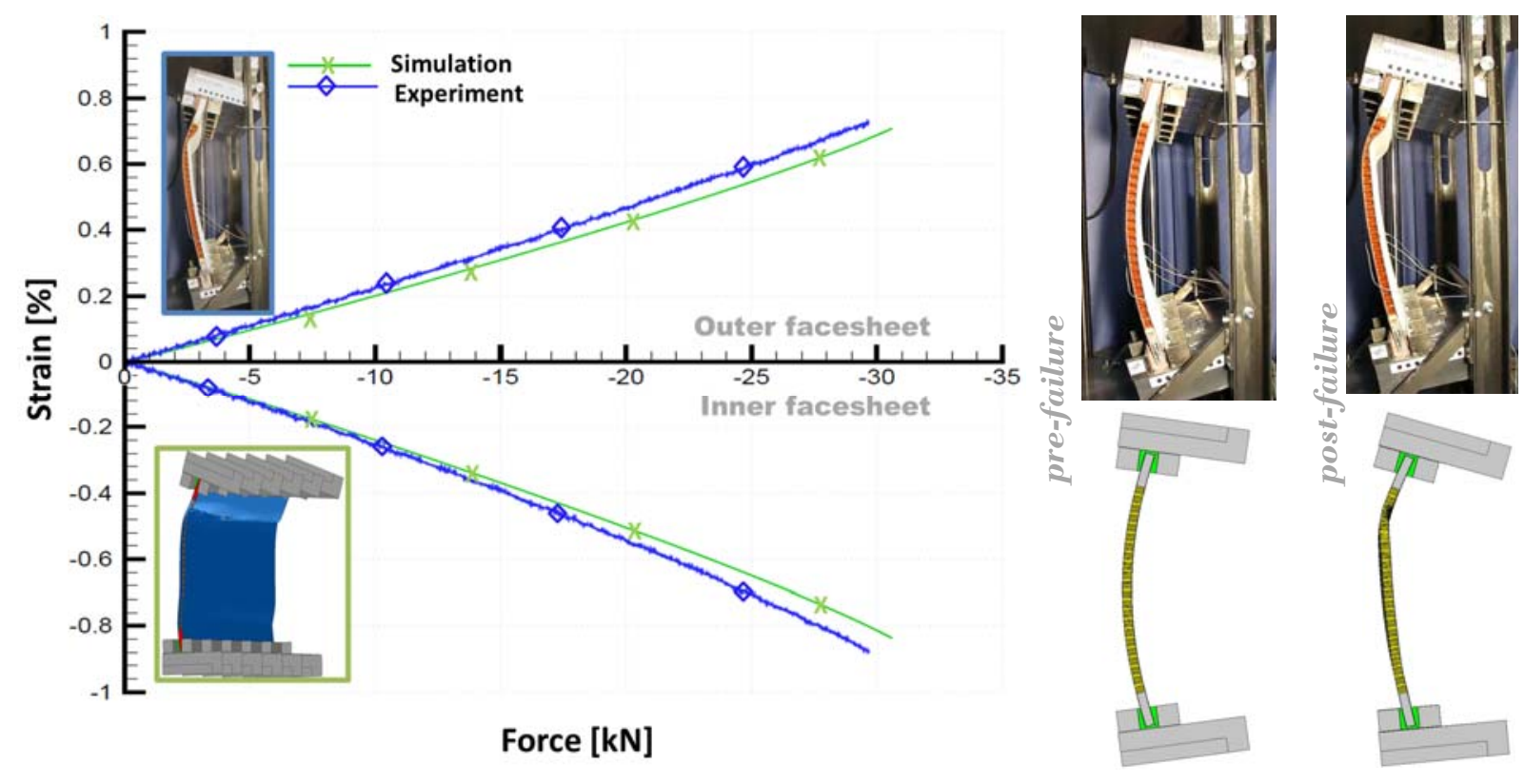

Fig. 8: Comparison between numerical and simulation with numerical representation of the edge imperfection of the untriggered foldcore panel under a bending-compression ratio of $200 \mathrm{~mm}$ 
The influence of the degraded fold edges does not considerably alter the stiffness of the panel since the correlation between simulation and experiment remains the same within the elastic domain. Hence the simulation quality of the post-failure domain could be significantly improved without noticeably affecting the elastic response of the sandwich panel. For further validation the experiment with the triggered sandwich panel was simulated $(A=200 \mathrm{~mm})$. The comparison between simulation and experiment is shown in Figure 9. By the integration of the weakened fold edges the correlation quality between simulation and experiment could be improved considerably. The simulation predicts a failure of the foldcore directly within the trigger region which corresponds to the experimental investigation. As in the experiment a complete fracture of the inner facesheet is obtained in the simulation.

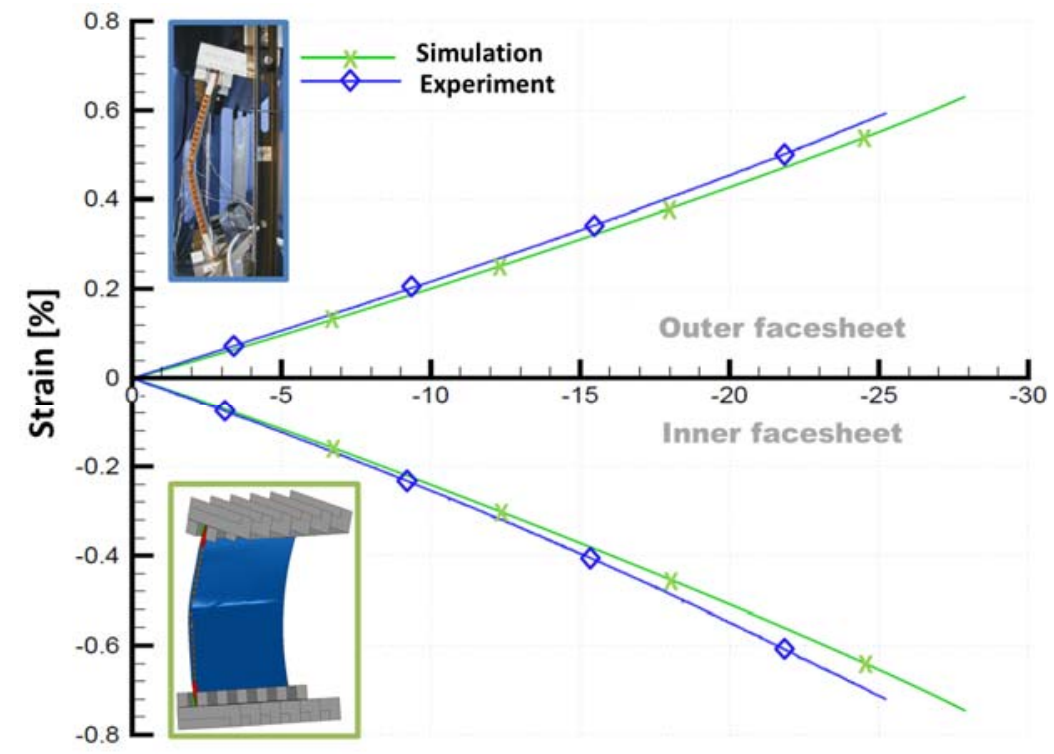

Force $[k N]$


Fig. 9: Comparison between numerical and simulation with numerical representation of the edge imperfection of the triggered foldcore panel under a bending-compression ratio of $200 \mathrm{~mm}$

A direct comparison of the strain-load curve between experiment and simulation shows that the elastic behaviour of the sandwich panel is not affected by the integration of the degraded fold edges. However, the simulation still predicts a considerable higher failure load. Though, in comparison to the investigation without the weakened fold edges the overestimation could be considerable reduced from $27 \%$ to only $12 \%$.

For further validation of the numerical methodologies a third component test of a triggered sandwich panel with reduced bending-to-compression ratio was investigated. Therefore, the eccentricity of the sandwich panel in regard to the loading path was reduced from $200 \mathrm{~mm}$ to $180 \mathrm{~mm}$. The results of the simulation and the experiment are plotted in Figure 10. The obtained result corresponds to the outcome of the simulation of the triggered panel with a bending-tocompression-ratio of $200 \mathrm{~mm}$. The simulation results for deformation and failure correlate to the experiment. As observed in the experiment the triggered core collapses which leads to a panel failure in this region. The direct comparison of the load-strain-curve shows the same tendency as obtained in the simulation with $A=200 \mathrm{~mm}$.

With regard to the numerical sensitivity of the exact failure prediction the presented numerical approach is able to give a quick understanding of the sandwich failure under various crash-relevant loading conditions. Additionally, the effect of a modified core pattern can be studied numerically without conducting an expensive test campaign. 

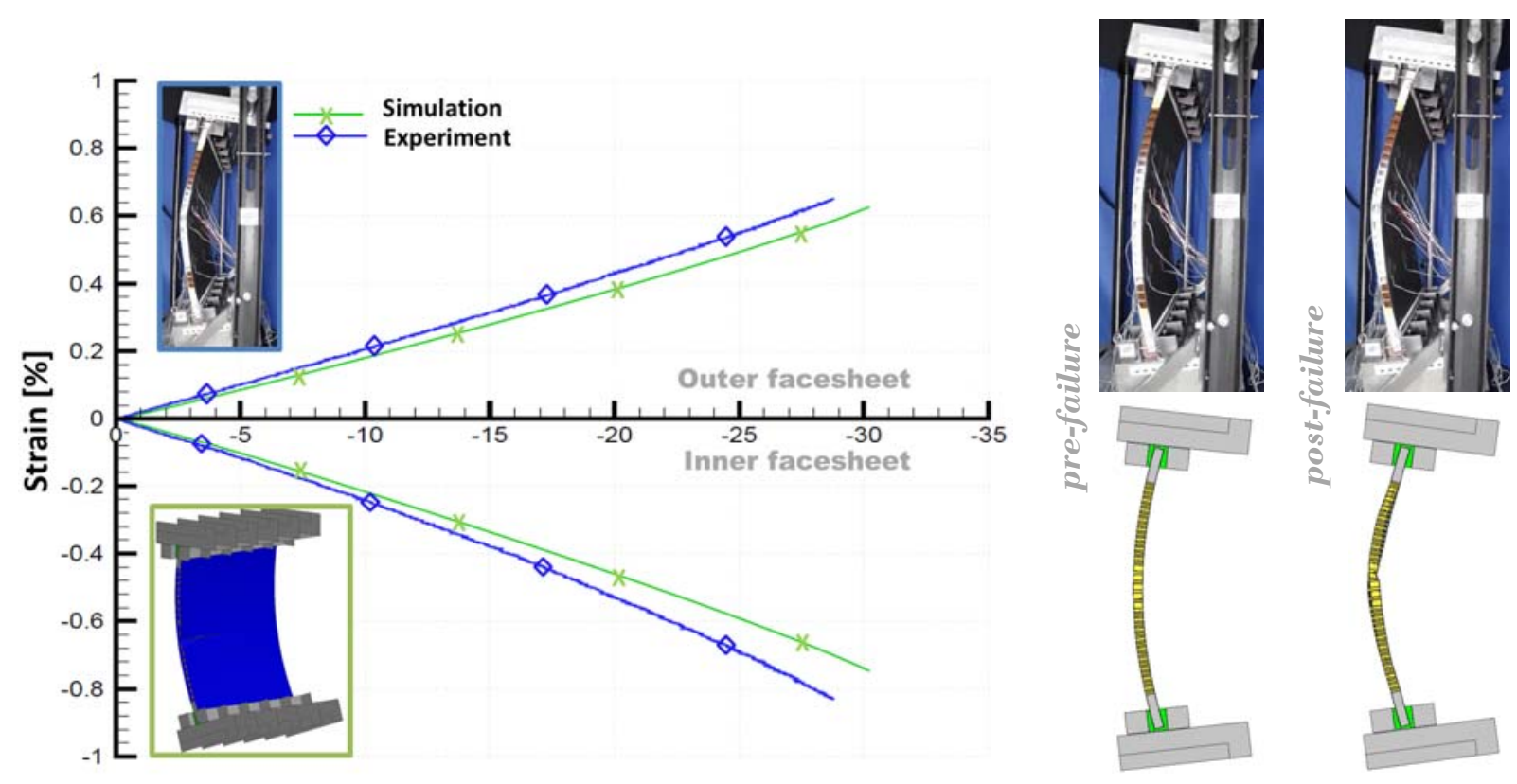

\section{Force $[k N]$}

Fig. 10: Comparison between numerical and simulation with numerical representation of the edge imperfection of the triggered foldcore panel under a bending-compression ratio of $180 \mathrm{~mm}$

\subsection{Virtual testing simulation}

Since failure can considerably differ under dynamic loading conditions, the robustness of the core trigger mechanism was numerically investigated under different crash-relevant loading rates. The developed simulation approach was used to investigate the dynamic behaviour. The difference in the loading condition was obtained by a modification of the displacement controlled loading condition. The panel failure up to a final loading rate of $4 \mathrm{~m} / \mathrm{s}$ is depicted in Figure 11 which corresponds roughly to the crash-relevant loading rate of the fuselage segment between the cargo- and the vertical struts. The core trigger shows a robust failure initiation for all investigated loading rates. Comparing the exact loading during failure, there is a small decrease in trigger load over the investigated loading range. However, the decrease is only caused by numerical effects. The required faster acceleration of the load introduction devices lead to stronger inertial effects. Due to the increased oscillation the implemented numerical threshold for element degradation is reached earlier which leads to a reduced failure load, if considering the filtered numerical output. According to numerical studies foldcore can show load-rate dependent compression behaviour due to changes in the buckling modes [15]. Since for all investigated loading rates the simulation predicts the same global buckling failure of foldcore in the trigger region, the rate dependent behaviour seems to be of lower significance for crash than for impact events, where the impact speed can differ significantly for the various impact hazards.

\begin{tabular}{c|l|l|l|} 
& $2 \mathrm{~m} / \mathrm{s}$ & $3 \mathrm{~m} / \mathrm{s}$ & $\mathbf{4} \mathrm{m} / \mathrm{s}$ \\
\hline $\begin{array}{c}\text { Force } \\
{[\mathrm{kN}]}\end{array}$ & $-\mathbf{3 3 , 2} \mathbf{~ k N}$ & $-\mathbf{3 1 , 9} \mathbf{~ k N}$ & $\mathbf{- 3 1 , \mathbf { 1 } \mathbf { ~ N }}$ \\
\hline $\begin{array}{c}\text { Strain } \\
{[\%]}\end{array}$ & $-\mathbf{0 , 9} \%$ & $-\mathbf{0 , 8 5} \%$ & $\mathbf{- 0 , 8 1} \mathbf{\%}$ \\
\hline
\end{tabular}
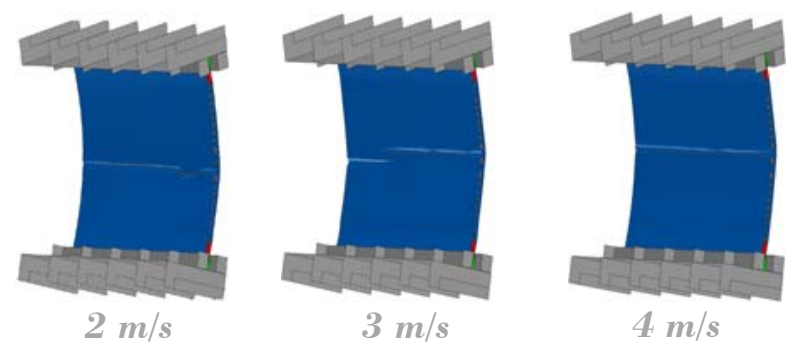

Fig. 11: Core trigger robustness for different loading rates 
Beside the failure under closing hinge conditions (the curvature of the panel increases), controlled failure initiation is also required under open hinge conditions (the curvature of the panel decreases) if a pre-defined crash kinematics for CFRP fuselage has to be obtained. Using the developed numerical methodology, the core trigger was also investigated under opening moment loads. Therefore, the panel was turned to obtain the required panel loading. Besides the different position of the panel, all remaining parameters were kept equal. In Figure 12 the structural behaviour of the triggered panel under open moment loading is shown. In the simulation the panel flattens until a failure at the load introduction is obtained. The structural behaviour is understandable since the maximum bending load and the known stress concentration near the load introduction are superimposed at the position of failure. Therefore, according to the simulation, trigger mechanisms are difficult to investigate under open loading condition using the current experimental technique.

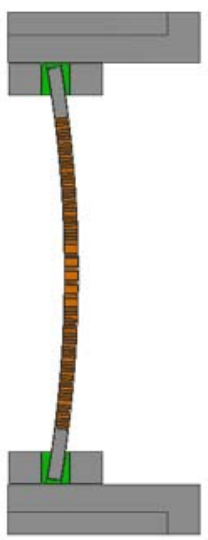

unloaded

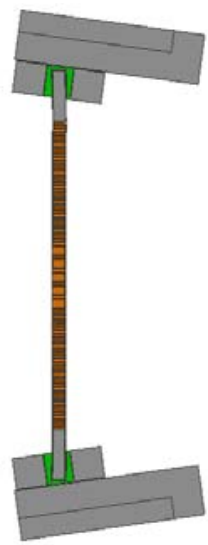

pre-failure

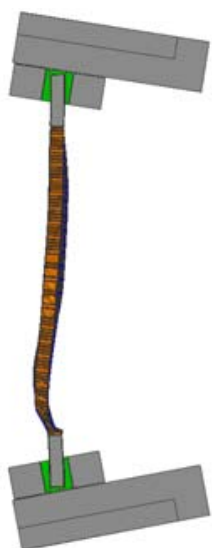

post-failure

Fig. 12: Identification of the core trigger functionality for open hinge applications

\section{Conclusions}

In the presented study the failure mechanisms of twin-walled fuselage panels with foldcore were investigated numerically under crash relevant compression-bending loads using an explicit simulation approach with a detailed core micromodel. The numerical results were validated by experiments investigating the panel failures under defined combined loading conditions. Despite the fact that the used material model for the aramid paper was successfully validated under various impact conditions [15], the known slight overestimation in the peak force considerably altered the numerical outcome in comparison to the experiments. This is different to the simulation of impact damage where the correlation quality strongly depends on the modelling of the overall energy absorption during failure, the exact prediction of the failure initiation is the main defining parameter for the virtual design of trigger mechanisms under crash loads. Since the identified overestimation in the failure strength is assumed to be originated by the idealised numerical representation of the foldcore material, the weakened fold edges were integrated into the simulation model. After including the degraded fold edges in the simulation model all experimentally obtained failure modes could be simulated. With the obtained grade of correlation quality the design process of folded sandwich panels can be supported by the presented virtual design methodology. 


\section{Acknowledgements}

The research leading to these results has received funding from the Helmholtz Association of German Research Centres. The authors gratefully acknowledge the funding of the research activities.

\section{References}

[1] Kindervater Ch., Thomson R., Johnson A., David M., Joosten M. et al., Validation of Crashworthiness Simulation and Design Methods by Testing of a Scaled Composite Helicopter Frame Section, AHS Conference, 2011

[2] Jackson A, Dutton S, Gunnion AJ, Kelly D., Effect of Manufacture and Laminate Design on Energy Absorption of Open Carbon-Fibre/Epoxy Sections, 17th Int. Conference on Composite Materials (ICCM-17), 2009

[3] Waimer M., Development of a Kinematics Model for Assessment of Global Crash Scenarios of a Composite Transport Aircraft Fuselage, PhD thesis, University of Stuttgart, ISRN DLR-FB-2013-28, 2013

[4] Schatrow P., Waimer M., Investigation of an Alternative Crash Concept for Composite Transport Aircraft using Tension Absorption, Seventh Triennial International Aircraft Fire and Cabin Safety Research Conference, 2013

[5] Pérez J. G., Energy Absorption and Progressive Failure Response of Composite Fuselage Frames, Master thesis, Virginia Polytechnic Institute, 1999

[6] Heimbs S., Hoffmann M., Waimer M., Schmeer S.,Blaurock J., Dynamic Testing and Modelling of Composite Fuselage Frames and Fasteners for Aircraft Crash Simulations, International Journal of Crashworthiness, Vol. 18, No. 4, 2013, pp. 406-422

[7] Waimer M., Kohlgrüber D., Hachenberg D., Voggenreiter H., The Kinematics Model - A Numerical Method for the Development of a Crashworthy Composite Fuselage Design of Transport Aircraft, Sixth Triennial International Aircraft Fire and Cabin Safety Research Conference, 2010

[8] EMIR, "Engineering mehrfunktionaler Integralstrukturen CfK-Rumpf", LuFo III (German national aeronautics research project), BMBF, (2003-2007).

[9] Drechsler K., Manufacturing of folded core-structures for technical applications, 25th International SAMPE Europe Conference, 508-513, 2004

[10] Kehrle R., Kolax M., Sandwich structures for advanced next generation fuselage concepts, SAMPE Europe Technical Conference, France, 2006

[11] Heimbs S., Middendorf P., Kilchert S., Johnson A., Maier M., Experimental and Numerical Analysis of Composite Folded Sandwich Core Structures Under Compression, Applied Composite Materials, Vol. 14, No. 5-6, 2007, pp. 363-377

[12] Klett Y., Auslegung multifunktionaler isometrischer Faltstrukturen für den technischen Einsatz, PhD thesis, University of Stuttgart, ISBN 978-3843910255, 2013 
[13] Kintscher M., Kärger L., Wetzel A., Hartung D., Stiffness and Failure behaviour of folded sandwich cores under combined transverse shear and compression, Composite Part A 38 (2007) 1288-1295, 2007

[14] Fischer S., Numerische Simulation der mechanischen Eigenschaften von Faltkern-Sandwichstrukturen, PhD thesis, University of Stuttgart, ISBN 978344012583, 2012

[15] Kilchert S., Nonlinear finite element modelling of degradation and failure in folded core composite sandwich structures, PhD thesis, University of Stuttgart, ISRN DLR-FB-2013-22, 2013

[16] Sturm R., Impactsimulation auf Rumpfschalen mit gefalteter Kernstruktur, German Aerospace Centre [DLR], Internal Report, DLR-IB 435-2005/22

[17] Heimbs S., Sandwichstrukturen mit Wabenkern: Experimentelle und numerische Analyse des Schädigungsverhaltens unter statischer und kurzzeitdynamischer Belastung, PhD thesis, Kaiserslautern University of Technology, 2008

[18] Nguyen M.Q., Jacombs S., Thomson R., Hachenberg D., Scott M.L., Simulation of impact on sandwich structures, Composite Structures 67, 2005

[19] Johnson A., Pentecôte N., Modelling Impact Damage in Double-Walled Composite Structures, VIII International Conference on Computational Plasticity, Barcelona, 2005

[20] R. Sturm, Klett Y. , Kindervater Ch., Voggenreiter H., Failure of CFRP airframe sandwich panels under crash-relevant loading conditions, Composite Structures, Vol. 112, pp. 11-21, 2014

[21] R. Sturm, Y. Klett, Kerntriggerung durch gezielte lokale Faltkernmodifikation, patent 102009047 040, Germany, 2012

[22] Ladeveze P., Inelastic strains and damage, Chapt 4 in Damage mechanics of composite materials, Composite Materials Series 9, Elsevier, Amsterdam, 1994

[23] Kilchert S., Johnson A., Voggenreiter H., Finite element modelling of phenolic resin impregnated aramid paper adopted in foldcore sandwich structures, CST 2008, Civil-Comp Press, paper 316, 2008

Ralf Sturm

1) *) Ralf Sturm

Deutsches Zentrum für Luft- und Raumfahrt e. V.

Pfaffenwaldring 38-40

70569 Stuttgart 